\title{
Cyclic fatigue resistance of two nickel-titanium instruments in different curving angles: a comparative study
}

\author{
Cheng PENG ${ }^{(b)}$ \\ Hui WU(b) \\ Lei WANG(b) \\ Xin $\mathrm{HU}^{(\mathrm{b})}$ \\ Shu DENG(a) \\ Changyi LI(b) \\ Lianyun ZHANG(b) \\ (a) Tianjin Medical University, Second Hospital \\ of Tianjin Medical University, Department of \\ Stomatology, Tianjin, China. \\ (b) Tianjin Medical University, School of \\ Stomatology, Tianjin, China.
}

Declaration of Interests: The authors certify that they have no commercial or associative interest that represents a conflict of interest in connection with the manuscript.

Corresponding Author:

Lianyun Zhang

E-mail: lianyun_zhang@163.com

DOI: 10.1590/1807-3107BOR-2015.vol29.0122

Submitted: Mar 19, 2015

Accepted for publication: Jun 30, 2015

Last revision: Aug 31, 2015

\begin{abstract}
The cyclic resistance of ProTaper Universal (size 25/08) and ProTaper Next (size 25/06) instruments was compared in artificial canals with different curvatures in this study. A total of 30 ProTaper Universal and 30 ProTaper Next instruments were divided into 6 groups $(n=10)$ and were operated into artificial canals with 3 different angles of curvature $\left(45^{\circ}, 60^{\circ}, 90^{\circ}\right)$. The canal length was kept consistent in this study. The number of cycles to fracture (NCF) was counted until file fracture occurred, at which point, the length of the fragment was measured. The data were analyzed statistically using ANOVA complemented by the Tukey test $(\mathrm{p}<0.05)$. Cross sections of the fractured files were scanned by an electron microscope. In the fatigue test, the ProTaper Next displayed more resistance in $45^{\circ}$ and $60^{\circ}$ canals $(\mathrm{p}<0.05)$, whereas ProTaper Universal exhibited a better operability in $90^{\circ}$ canals $(\mathrm{p}<0.05)$. The average length of the fragments from ProTaper Next was significantly shorter than that from ProTaper Universal in $90^{\circ}$ canals $(\mathrm{p}<0.05)$. The cross sections of the fractured surfaces became flatter when the curvature angles decreased from $90^{\circ}$ to $45^{\circ}$. ProTaper Next was more reliable when shaping in curved canals, whereas ProTaper Universal was more sui for the preparation of root canals with severe curvatures.
\end{abstract}

Keywords: Fractures, Stress; Root Canal Therapy; Dental Alloys.

\section{Introduction}

Files are the major instruments in root canal therapy. A perfect file should be flexible and have a high cutting efficiency, which can both prevent file fracture inside the canals and guarantee the initial shapes of the canals. Nickel-titanium (NiTi) instruments, which were first introduced by Walia et al., ${ }^{1}$ have become popular in recent years because NiTi files display more flexible than steel ones. However, the fracture of NiTi files remains a challenge for clinicians.

The repeated cyclic fatigue caused by the instrumentation of curved canals may be the crucial factor in instrument fracture. The fracture of rotary NiTi files can be broadly classified into 2 types: torsional fatigue fracture and flexural (cyclic) fatigue fracture. Torsional fatigue usually occurs when the tip or some part of a file binds in the root canal but the shaft still rotates. Flexural fatigue is caused by repeated compressive and tensile forces accumulating around the maximally 
curved point of files. When shaping a curved canal, a file is subjected to compressive stress on the inside of the shaft and to tensile stress along the outside of the shaft. Every portion of the rotated file suffers from continuous compressive and tensile stress. Microfissures and microfatigue are generated on the surface of files during this process. Thus, a sudden fracture can occur when stress accumulates around these regions. ${ }^{2}$ No warning of fracture progress can be observed because the microfissure and microfatigue stays invisible, even to microscope analysis. ${ }^{3}$ According to the work of Yared et al., ${ }^{4} \mathrm{NiTi}$ rotary instruments can be safely used in simulated tests up to 10 times. Knowing when to abandon a file is pragmatic for clinicians. Therefore, understanding the resistance of cyclic fatigue is meaningful for clinicians during the process of shaping curved canals.

Simulated testing has become the most popular way to explore the fatigue characteristics of files after the first device for testing cyclic fatigue resistance was introduced by Gambarini ${ }^{5}$ and Gambarini et al..$^{6}$ Several authors have compared the influence of the curvature radius and the curvature angle on the number of cycles to fracture (NCF) by performing cyclic fatigue tests, ${ }^{7,8}$ but these designs were questionable because when the radius of curvature was kept constant, increasing the curvature angles could lead to the shortening of the arch length. Discrepancies in parameters such as the radius, angle of curvature and arch length contribute to the different levels of fatigue resistance of various files. In this study, arches with a standard length and different curvature angles were positioned at the end of each artificial canal to simulate the shape of a single root canal.

ProTaper Next, which was manufactured by M-Wire, was introduced recently. This system has an off-centered rectangular cross-section design. Files that are manufactured with the M-wire method showed more flexible and fatigue resistance than did conventionally manufactured files. ${ }^{9}$ The design of the cross section enhances the resistance to stress and increases the efficiency of shaping due to its unique asymmetric rotary motion. ${ }^{10}$ ProTaper Universal was manufactured conventionally with a triangle section. The improved designs should facilitate the cyclic fatigue resistance of the files, but no reports comparing the cyclic fatigue resistance between these types of files have been reported yet. Although the point of the maximum stress has been reported to be the midpoint of the curving shaft, the relationship between the breakage point on the file shaft and the curving angle has not yet been reported. Therefore, the aim of this study was to compare the fatigue resistance of ProTaper Next and ProTaper Universal in different curvature angles and to analyze the position change of the breakage points on the file shaft when the angle of the curved canal changes.

\section{Methodology}

ProTaper Universal (DentsplyMaillefer, Ballaigues, Switzerland) and ProTaper Next (DentsplyMaillefer, Ballaigues, Switzerland) were tested in this study. In this manuscript, PT Universal is short for ProTaper Universal, and PT Next is short for ProTaper Next. The instruments were divided as follows: PT F2 $(25 \mathrm{~mm}$ in length, $\mathrm{n}=30$ ) and PTN X2 (25 mm in length, $\mathrm{n}=30$ ). An apparatus was designed for simulating different curved canals. The apparatus was similar to the one designed by Sterling J. Whipple, which included 3 cylinders and 3 matching jigs. ${ }^{11}$ Each cylinder had 3 grooves ( $1.5 \mathrm{~mm}$ wide and $1 \mathrm{~mm}$ deep). The radii of the cylinders were $12 \mathrm{~mm}, 9 \mathrm{~mm}$ and $6 \mathrm{~mm}$ (Figures 1, 2 and 3). The matching jigs had a vertical palate ( $10 \mathrm{~mm}$ length), and all were extended in arches with the same length $(9.42 \mathrm{~mm})$ but with different radii $(12 \mathrm{~mm}, 9 \mathrm{~mm}$ and $6 \mathrm{~mm})$, respectively. The angles of curvature of the 3 jigs were $45^{\circ}, 60^{\circ}$ and $90^{\circ}$, and the radii of the cylinders were $12 \mathrm{~mm}$, $9 \mathrm{~mm}$ and $6 \mathrm{~mm}$, respectively. The artificial canal was demarcated by the space surrounded by the jig and the slot in the cylinder. The 3 canals consisted of 3 slots on each cylinder and the matching jigs. The initial distance between the jig and the groove was $2 \mathrm{~mm}$, and the artificial canals tapered to $1 \mathrm{~mm}$ at the end of the arch.

The position of the electronic handpiece (Smart plus, DensplyMaillefer Ballaigues, Switzerland) was controlled by an arm fixed to the apparatus. The handpiece could move up and down by splinting the screw. 


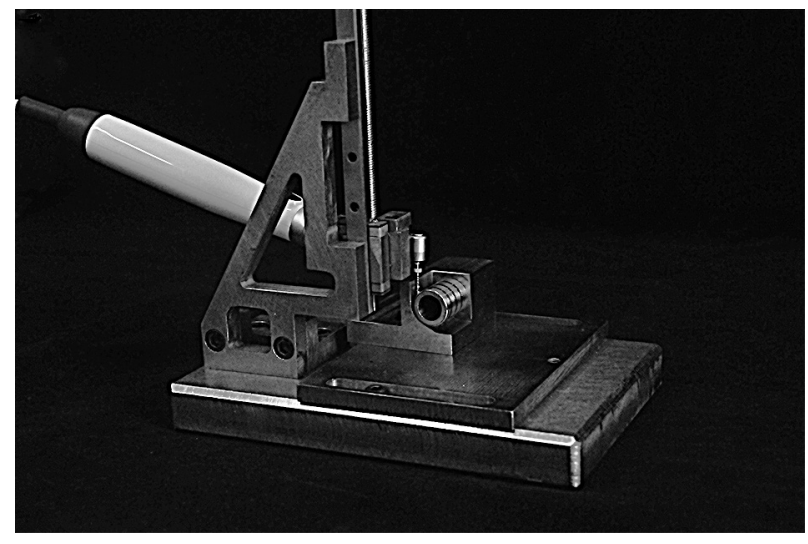

Figure 1. The apparatus with the file inserted into the artificial canals.

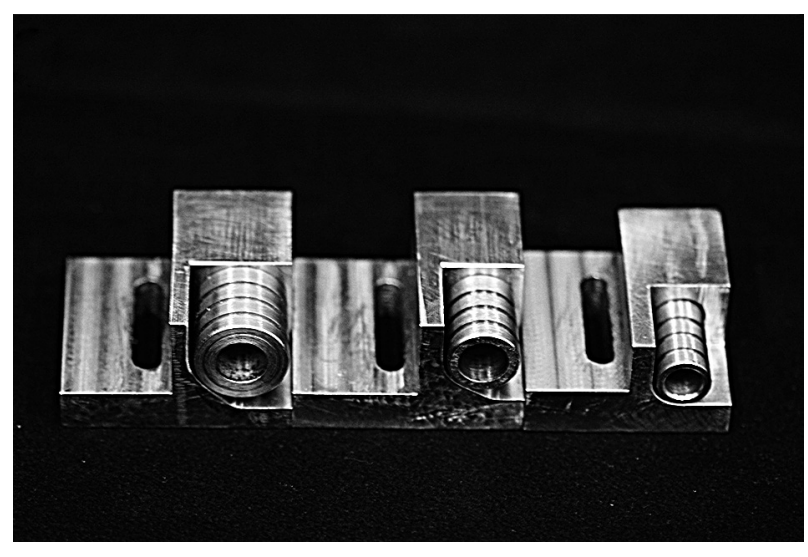

Figure 2. A photo of the apparatus in cross-section.

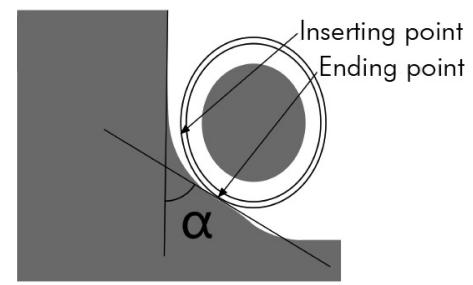

*The file was inserted from an insertion point $2 \mathrm{~mm}$ wide and ending at a $1 \mathrm{~mm}$ wide point. The angle of curvature $\alpha$ included the $45^{\circ}, 60^{\circ}$ and $90^{\circ}$ canals.

Figure 3. A schematic of the apparatus in cross-section.

In this study, we set up six groups as follows: PT Universal operated in the $45^{\circ}$ canal (group A, $n=10$ ), PT Universal operated in the $60^{\circ}$ canal (group B, $\mathrm{n}=10$ ), PT Universal operated in the $90^{\circ} \mathrm{canal}$ (group $\mathrm{C}, \mathrm{n}=10$ ), PT Next operated in the $45^{\circ}$ canal (group D, $\mathrm{n}=10$ ), PT Next operated in the $60^{\circ}$ canal (group E, $\mathrm{n}=10$ ) and PT Next operated in the $90^{\circ}$ canal (group
$\mathrm{F}, \mathrm{n}=10$ ). The rotating speed was $250 \mathrm{rpm}$ with a torque of 2 N.cm. The files rotated freely without friction with the aid of a special oil (WD-40, Milton Keynes, England) $)^{12}$, and air spray was used to avoid the accumulation of heat. ${ }^{13}$ The working time was recorded with a digital stop watch (Omega, 1/100 s chronometer, Bienne, Switzerland) accurate to 0.01 second. The NCF was counted when the fracture occurred. The length of fragments was calculated by subtracting the rest length from the initial length. The cross sections of the fractured files were observed with an electron microscope (HITACHI 4800, HITACHI, Tokyo, Japan) to observe the features of the fracture.

Statistical analysis of the NCF and length of fragments were analyzed by an independent-sample $\mathrm{T}$ Test or 2-independent Samples Nonparametric Test according to the homogeneity or heterogeneity of variance, as calculated by a one-way analysis of variance (ANOVA). All data were analyzed by software (SPSS 19.0; Chicago, USA) and the significance was set at $\mathrm{p}<0.05$.

\section{Results}

PT Next exhibited better resistance to cyclic fatigue than PT Universal when operated in $45^{\circ}$ and $60^{\circ}$ canals, but PT Universal had a better operability in the $90^{\circ}$ canal, as displayed in Table 1. Similar to these products, the NCF of files from groups operated in the $45^{\circ}$ canal ranked the first, and files operated in the $90^{\circ}$ canal ranked third by a significant margin, although no significant difference was found between Groups B and C.

The length of the fractured fragments from PT Next was significantly shorter than that from PT Universal when operated in the $90^{\circ}$ canal (Groups C and F). However, no significant difference was found between these groups when operated in the $45^{\circ}$ and $60^{\circ}$ canals. For the same file type, files operated in the $45^{\circ}$ canal (Groups A and D) resulted in significantly longer fragments than did the files operated in the $60^{\circ}$ canal (Groups B and E), respectively. No significant difference was found between the $60^{\circ}$ canal (Groups $\mathrm{B}$ and E) and the $90^{\circ}$ canal (Groups C and F). The results are displayed in Table 2 .

The cross sections of the fracture surface from Groups $\mathrm{C}$ and $\mathrm{F}$ are noted to be the most irregular, 
Table 1. Mean ( \pm SD) of the NCF $(n=10)$ for different groups.

\begin{tabular}{lccc}
\hline Groups & $45^{\circ}$ & $60^{\circ}$ & $90^{\circ}$ \\
\hline PT Universal & $\mathrm{A}: 1458( \pm 546)$ & $\mathrm{B}: 390( \pm 110)$ & $\mathrm{C}: 330( \pm 44)$ \\
PT Next & $\mathrm{D}: 4642( \pm 1314)$ & $\mathrm{E}: 524( \pm 60)$ & $\mathrm{F}: 220( \pm 22)$ \\
ANOVA/Turkey's Test & $\mathrm{p}=0.000$ & $\mathrm{p}=0.000$ & $\mathrm{p}=0.000$ \\
\hline
\end{tabular}

Table 2. Mean ( \pm SD) of the fractured fragments of different groups $(n=10)$.

\begin{tabular}{lccc}
\hline Groups & $45^{\circ}$ & $60^{\circ}$ & $90^{\circ}$ \\
\hline PT Universal & $\mathrm{A}: 2.47 \mathrm{~mm}( \pm 0.43)$ & $\mathrm{B}: 3.88 \mathrm{~mm}( \pm 0.53)$ & $\mathrm{C}: 4.40 \mathrm{~mm}( \pm 0.39)$ \\
PT Next & $\mathrm{D}: 2.52 \mathrm{~mm}( \pm 0.31)$ & $\mathrm{E}: 3.55 \mathrm{~mm}( \pm 0.55)$ & $\mathrm{F}: 3.82 \mathrm{~mm}( \pm 0.25)$ \\
ANOVA/Turkey's Test & $\mathrm{p}=0.791$ & $\mathrm{p}=0.069$ & $\mathrm{p}=0.000$ \\
\hline
\end{tabular}

and pits and crack regions can be observed on the cross sections. The cross sections became flat and regular when the canal angle decreased from $90^{\circ}$ to $45^{\circ}$, respectively. The fracture was first observed from the edge of the files (Figures 4, 5 and 6).

\section{Discussion}

File fracture is the main concern for clinicians during the process of root canal shaping. ${ }^{14}$ Cyclic fatigue has been reported to be one of the major factors resulting in file fracture in curved canals. ${ }^{15}$ The cyclic fatigue test has been reported to be a simple but reliable approach to reflect the fatigue behavior of the NiTi file. ${ }^{16,17}$ In the previous studies,

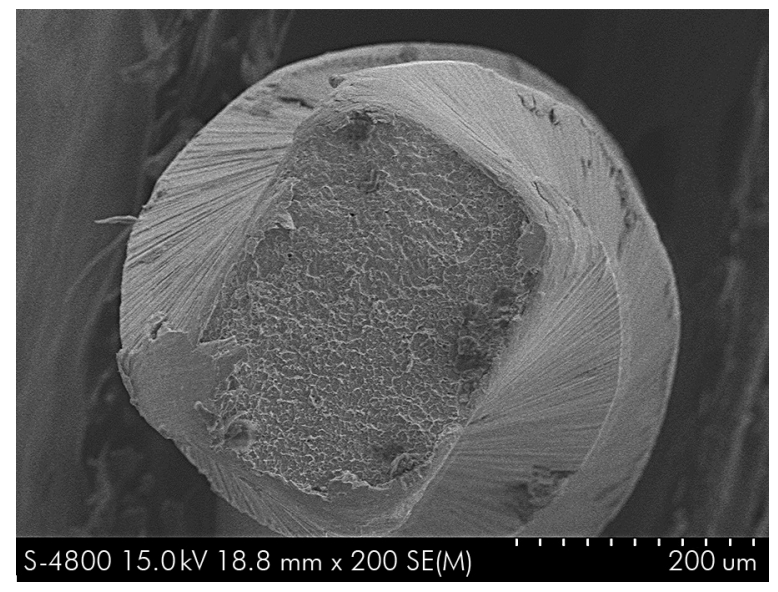

Figure 4. Scanning electron micrographs of the cross surface of the fractured ProTaper Next files from a $90^{\circ}$ canal.

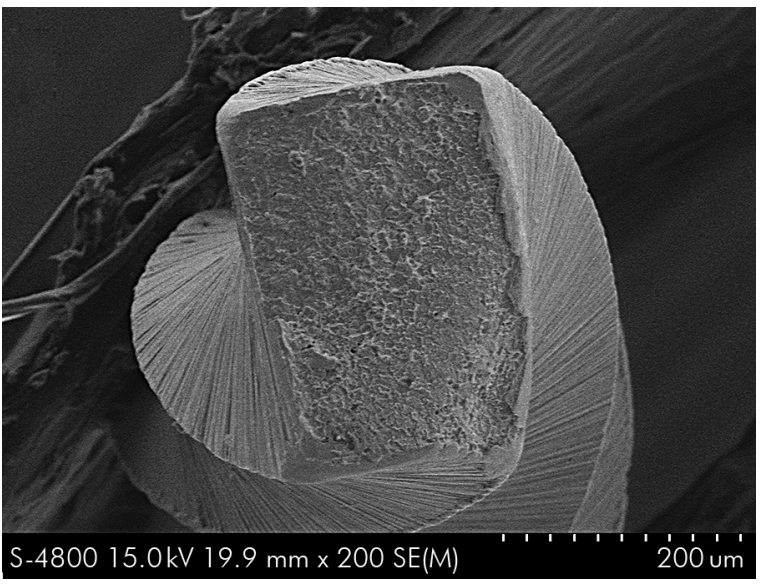

Figure 5. Scanning electron micrographs of the cross surface of the fractured ProTaper Next files from a $60^{\circ}$ canal.

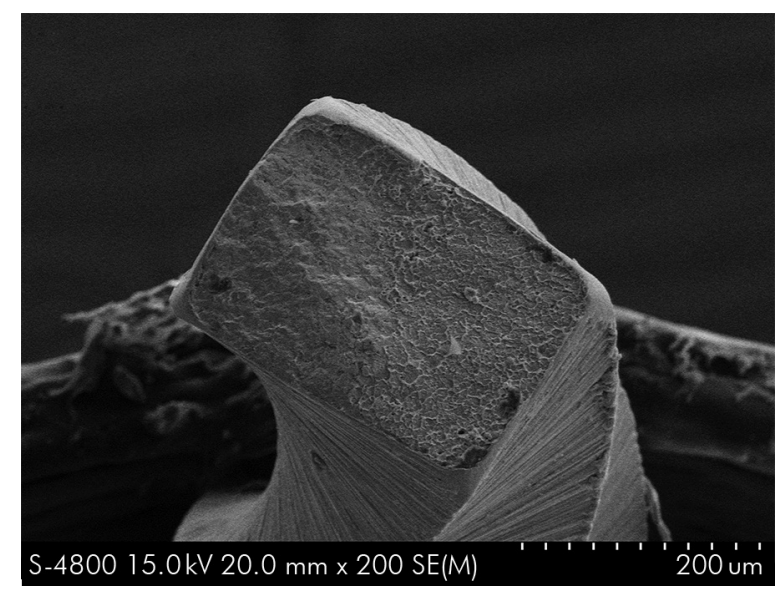

Figure 6. Scanning electron micrographs of the cross surface of the fractured ProTaper Next files from a $45^{\circ}$ canal. 
the characteristics of the curved canals, which could influence the fatigue resistance for files, included the angle of curvature ${ }^{18,19,20}$ and the radius. ${ }^{7,12,18,19,20,21}$ The angle of curvature (measured in degrees) is related to the length of the arc (measured in millimeters). Several authors have compared the influence of the radius length and the curvature angle on the NCF using cyclic fatigue tests. ${ }^{12,21}$. However, this comparison is questionable because changing the radius also changes the arch length when the curvature angles are kept constant. As the radius length increases, the NCF values of an instrument subjected to cyclic fatigue will increase. Conversely, shorter arch lengths displayed lower NCF values as the radius became shorter. It is impossible to determine which parameter is preponderant, as the radius, the curvature angle and the arch length also influence the resistance of the file. ${ }^{22}$ Thus, the length of the arch was controlled in this study to compare the influence of the curvature angle and the radius on the resistance to cyclic fatigue between the PT Universal and PT Next operated in different curvature angles and curvature radii.

The manufacturing process for Ni-Ti files can be different, and there are three phase transformations of Ni-Ti alloy during this process: Austenite, Martensite and R-phase. ${ }^{23}$ PT Next was manufactured by M-Wire. The mechanical characteristics of the Ni-Ti alloys were reported to be extremely sensitive to the microstructures associated with their thermomechanical treatment history, and the M-Wire contained all 3 of the above crystalline phases. ${ }^{24} \mathrm{M}$-Wire rendered the files more flexible and fatigue resistant than did the conventional $\mathrm{Ni}$-Ti files that are in clinical use..$^{25}$ The results of this study were consistent with these reports when the files were operated in $45^{\circ}$ and $60^{\circ}$ canals. The Ni-Ti Files with M-Wire had higher Vickers hardness values compared with the conventional ones reported by Zinelis et al. ${ }^{26}$ Thus, PT Next could be found to suffer from more comprehensive and tonsil stress when operated in curved canals. This stress increased as the angle of curvature increased.

The cross-sections of the PT Universal files were triangular, whereas the cross-sections of the PT Next files were off-centered and rectangular. The resulting asymmetric motion of the latter could increase the cutting efficiency of the PT Next, and the symmetric rotary motion might disperse the stress on the shaft of the PT Universal. Thus, increased stress levels led to PT Next fatigue, which caused PT Universal to be more fatigue resistant than PT Next in $90^{\circ}$ canals.

The fracture of Ni-Ti files caused by fatigue usually occurs around the apical third of the canal, in the area with the highest curvature. Our results were consistent with this finding. The breaking point on the shaft moved coronally, and the angle of curvature increased. However, no significant difference was found between the PT Universal and PT Next files when operated in the $45^{\circ}$ canals and $60^{\circ}$ canals. When operated in the $90^{\circ}$ canals, the PT Next files fractured at a lower position than did the PT Universal files.

The observations of the cross-sections scanned by the electron microscope displayed similar features between the types of files. The characteristic is consistent with the findings of previous studies. ${ }^{27}$ When the surface of the cross-section of the fractured file was flat, the cracks tended to be generated in the same plane. We detected fewer microfissures generated around the highest curvature point of the file, which resulted in flat surfaces. It was observed that more microfissures occurred around the breakage point of the files operated in severely curved canals. However, these microfissures propagated from different planes. In other words, more microfissures were generated when the angle of curvature was increased, which subsequently increased the possibility of file fracture.

The major step for a simulated test is to provide the space for free file rotation. The inside and outside walls are both essential, as the file will suffer tensile and compressive stresses when shaping a curved canal. In this study, the benefit of our device was that the artificial canals consisted of four walls. However, the distance between the outside and inside walls was slightly wider than the diameter of the file to prevent the file from getting stuck in the artificial canals, which would not allow the file rotation to precisely fit. This design can present the file from getting stuck inside the artificial canals using oil lubrication. In this case, no torsional fatigue was noted in the files. This finding is different from the files extruded in the dentin in clinical work. At the same time, many other factors, including the rotation speed, the design of the artificial canals, and the temperature increase, will all influence the results. ${ }^{16}$ 


\section{Conclusion}

In conclusion, PT Next files tended to be more resistant than PT Universal files when operated in $45^{\circ}$ and $60^{\circ}$ canals, although this finding did not hold for operation in $90^{\circ}$ canals. Considering that the majority of root canals are closer to being

\section{References}

1. Short JA, Morgan LA, Baumgartner JC. A comparison of canal centering ability of four instrumentation techniques. J Endod. 1997;23(8):503-7. doi:10.1016/S0099-2399(97)80310-X

2. Vieira EP, Franca EC, Martins RC, Buono VT, Bahia MG. Influence of multiple clinical use on fatigue resistance of ProTaper rotary nickel-titanium instruments. Int Endod J. 2008;41(2):163-72. doi:10.1111/j.1365-2591.2007.01336.x

3. Lopes HP, Moreira EJ, Elias CN, Almeida RA, Neves MS. Cyclic fatigue of ProTaper instruments. J Endod. 2007;33(1):55-7. doi:10.1016/j.joen.2006.09.003

4. Yared GM, Dagher FE, Machtou P. Cyclic fatigue of Profile rotary instruments after simulated clinical use. Int Endod J. 1999;32(2):115-9. doi:10.1046/j.1365-2591.1999.00201.x

5. Gambarini G, Grande NM, Plotino G, Somma F, Garala M, De Luca $\mathrm{M}$ et al. Fatigue resistance of engine-driven rotary nickel-titanium instruments produced by new manufacturing methods. JEndod. 2008;34(8):1003-5. doi:10.1016/j.joen.2008.05.007

6. Gambarini G. Cyclic fatigue of nickel-titanium rotary instruments after clinical use with low- and high-torque endodontic motors. J Endod. 2001;27(12):772-4. doi:10.1097/00004770-200112000-00015

7. Pruett JP, Clement DJ, Carnes DL Jr. Cyclic fatigue testing of nickel-titanium endodontic instruments. J Endod. 1997;23(2):77-85. doi:10.1016/S0099-2399(97)80250-6

8. Yao JH, Schwartz SA, Beeson TJ. Cyclic fatigue of three types of rotary nickel-titanium files in a dynamic model. J Endod. 2006;32(1):55-7. doi:10.1016/j.joen.2005.10.013

9. Shen Y, Cheung GS, Bian ZA, Peng B. Comparison of defects in ProFile and ProTaper systems after clinical use. J Endod. 2006;32(1):61-5. doi:10.1016/j.joen.2005.10.017

10. Elnaghy AM. Cyclic fatigue resistance of ProTaper Next nickel-titanium rotary files. Int Endod J. 2014;47(11):1034-9. doi:10.1111/iej.12244

11. Whipple SJ, Kirkpatrick TC, Rutledge RE. Cyclic fatigue resistance of two variable-taper rotary file systems: ProTaper universal and V-Taper. J Endod. 2009;35(4):555-8. doi:10.1016/j.joen.2009.01.002

12. Inan U, Aydin C, Tunca YM. Cyclic fatigue of ProTaper rotary nickel-titanium instruments in artificial canals with 2 different radii of curvature. Oral Surg Oral Med Oral Pathol Oral Radiol Endod. 2007;104(6):837-40. doi:10.1016/j.tripleo.2007.06.019 $45^{\circ}$ or $60^{\circ}$ artificial canals, PT Next files should have a more widespread use in clinical applications. According to the observed resistance of the PT Universal files when operated in the $90^{\circ}$ canals, the PT Universal files can be used in canals with severe curvature.

13. Plotino G, Grande NM, Cordaro M, Testarelli L, Gambarini G. A review of cyclic fatigue testing of nickel-titanium rotary instruments. J Endod. 2009;35(11):1469-76. doi:10.1016/j.joen.2009.06.015

14. Kim HC, Yum J, Hur B, Cheung GS. Cyclic fatigue and fracture characteristics of ground and twisted nickel-titanium rotary files. J Endod. 2010;36(1):147-52. doi:10.1016/j.joen.2009.09.037

15. Sattapan B, Nervo GJ, Palamara JE, Messer HH. Defects in rotary nickel-titanium files after clinical use. J Endod. 2000;26(3):161-5. doi:10.1097/00004770-200003000-00008

16. Lopes HP, Ferreira AA, Elias CN, Moreira EJ, Oliveira JCM, Siqueira Junior JF. Influence of Rotational Speed on the Cyclic Fatigue of Rotary Nickel-Titanium Endodontic Instruments. J Endod. 2009;35(7):1013-6. doi:10.1016/j.joen.2009.04.003

17. Tobushi H, Shimeno $Y$, Hachisuka T, Tanaka K. Influence of strain rate on superelastic properties of TiNi shape memory alloy. Mech Mater. 1998;30(2):141-50. doi:10.1016/S0167-6636(98)00041-6

18. Zelada G, Varela P, Martin B, Bahillo JG, Magan F, Ahn S. The effect of rotational speed and the curvature of root canals on the breakage of rotary endodontic instruments. J Endod. 2002;28(7):540-2. doi:10.1097/00004770-200207000-00014

19. Kitchens GG Jr, Liewehr FR, Moon PC. The effect of operational speed on the fracture of nickel-titanium rotary instruments. J Endod. 2007;33(1):52-4. doi:10.1016/j.joen.2006.09.004

20. Pirani C, Cirulli PP, Chersoni S, Micele L, Ruggeri $\mathrm{O}$, Prati C. Cyclic fatigue testing and metallographic analysis of nickel-titanium rotary instruments. J Endod. 2011;37(7):1013-6. doi:10.1016/j.joen.2011.04.009

21. Grande NM, Plotino G, Pecci R, Bedini R, Malagnino VA, Somma F. Cyclic fatigue resistance and three-dimensional analysis of instruments from two nickel-titanium rotary systems. Int Endod J. 2006;39(10):755-63. doi:10.1111/j.1365-2591.2006.01143.x

22. Lopes HP, Vieira MV, Elias CN, Goncalves LS, Siqueira JF Jr, Moreira EJ et al. Influence of the geometry of curved artificial canals on the fracture of rotary nickel-titanium instruments subjected to cyclic fatigue tests. J Endod. 2013;39(5):704-7. doi:10.1016/j.joen.2012.12.027

23. Thompson SA. An overview of nickel-titanium alloys used in dentistry. Int Endod J. 2000;33(4):297-310. doi:10.1046/j.1365-2591.2000.00339.x 
24. Ye J, Gao Y. Metallurgical characterization of M-wire nickel-titanium shape memory alloy used for endodontic rotary instruments during low-cycle fatigue. J Endod. 2012;38(1):105-7. doi:10.1016/j.joen.2011.09.028

25. Shen Y, Zhou HM, Zheng YF, Peng B, Haapasalo M. Current challenges and concepts of the thermomechanical treatment of nickel-titanium instruments. J Endod. 2013;39(2):163-72. doi:10.1016/j.joen.2012.11.005
26. ZinelisS, Eliades T, Eliades G. A metallurgical characterization of ten endodontic Ni-Ti instruments: assessing the clinical relevance of shape memory and superelastic properties of Ni-Ti endodontic instruments. Int Endod J. 2010;43(2):125-34 doi:10.1111/j.1365-2591.2009.01651.x

27. Shen Y, Qian W, AbtinH, Gao Y, Haapasalo M. Fatigue testing of controlled memory wire nickel-titanium rotary instruments. J Endod. 2011;37(7):997-1001. doi:10.1016/j.joen.2011.03.023 